\title{
Relationship between the anteroposterior diameter of the patellar tendon. Pain and functionality in volleyball players
}

\author{
Calvo Gonell A. ${ }^{1}$ Macia Soler L. ${ }^{2}$ Moncho J. ${ }^{3}$ \\ ${ }^{1}$ Phsyiotherapy center, Castellón, Spain \\ 2 Department of Nursing. Faculty of Health Sciences. Universidad de \\ Alicante, Alicante, Spain \\ ${ }^{3}$ Department of Community Nursing, Preventive medicine and Public \\ Health and History of Science. Universidad de Alicante, Alicante, \\ Spain
}

Rev Fisioter Invasiva 2019;2:67.

\begin{abstract}
Keywords

- volleyball

- patellar tendon

- sports injuries

Introduction and Aim Overuse injuries in volleyball represent between $50 \%$ and $80 \%$ of total injuries, of which, $80 \%$ are patellar tendinopathies. Volleyball is the sport with the greatest prevalence of patellar tendinopathy, estimated at $44.6 \%$, followed by basketball with $31.9 \%$. The tendon thickness bears a close and direct relationship with abnormal and diffuse images with accumulation of ground substance, and affecting both sexes, although less marked in women.

Aim To determine whether the antero-posterior diameter of the patellar tendon in volleyball players is associated with a greater sensation of pain and reduced functionality.

Material and Methods An observational, descriptive, cross-sectional study on volleyball players. The scores on the VISA-P scale, the visual analog scale (VAS) and, using musculoskeletal ultrasound, the anteroposterior diameter of the patellar tendon at 5 and $10 \mathrm{~mm}$ distal to the inferior pole of the patella. Multiple linear regression models were constructed to adjust the effect of the anteroposterior diameter on the VAS and the VISA-P scores.

Results The final sample comprised 112 players. The anteroposterior diameter was greater in men and was significantly associated with scores on the VAS and the VISA-P for both tendons, and therefore, the greater the score of the diameter, the greater the score on the VAS and the lesser the score on the VISA-P.

Conclusions The measurement of the antero-posterior diameter of the patellar tendon using musculoskeletal ultrasound may be useful for the prevention of sports injuries in volleyball players, as it is significantly associated with a greater perception of pain and reduced functionality.
\end{abstract}

